\title{
A NEW APPROACH TO THE MECHANICS OF ORTHOTROPIC MULTILAYERED PLATES $\dagger$
}

\author{
M. A. BIOT
}

Avenue Paul Hymans 117, 1200 Brussels, Belgium

\begin{abstract}
New methods are developed which provide a simplified treatment of the mechanics of multilayered and strongly orthotropic plates. It includes the case where individual layers are themselves constituted by thinly laminated materials each with their own orthotropic and microelastic properties. The fundamental "skin effect" of anistropic solid mechanics is taken into account. The theory is derived in the context of plane strain deformations. Recurrence equations are obtained for an arbitrary number of layers and applied to plates with two and three layers. The microelastic stress-couple is evaluated for the case of laminated media. Characteristic physical features are discussed and the results are compared to a treatment based on the classical Euler-Bernouilli and Timoshenko models.
\end{abstract}

\section{INTRODUCTION}

COMPOSITE structures constituted by multilayered materials have come into extensive use in technology. This includes the use of thinly layered laminated materials whose bulk properties are strongly anisotropic with a microelastic bending rigidity. Exact methods of analysis of the dynamic properties of these structures by the theory of elasticity are in most cases impractical for usual design purpose. On the other hand, the classical "strength of materials" approach based on the Euler-Bernouilli or the Timoshenko beam model is not adequate. These classical models ignore the skin effect as analyzed and described earlier [1] which constitutes a fundamental feature in the mechanics of anisotropic solids. Hence they cannot predict the detailed stress-field distribution which is essential for the evaluation of local failures and overall damping capacity. In addition, the classical models do not take into account the cross-sectional distortion which plays an important role in the coupling of adherent layers. The present theory is an attempt to provide an approximate treatment which is much simpler than the one derived from elasticity theory while retaining the essential features. It is applicable to very complex structures with many layers such that the individual layers themselves may be constituted by thinly laminated materials with strongly orthotropic and microelastic properties.

It should be pointed out that the advantages resulting from a simplified theory are not only computational. They are also conceptual, due to a better understanding of the physical factors involved, leading to design improvements. An important characteristic feature brought out by the analysis is represented by the concept of "transition wavelength" which marks the point where the skin effect becomes significant.

For comparison, reference should be made to the rigorous analysis of multilayered media based on the theory of elasticity with initial stress as developed several years ago

$\dagger$ This research has been sponsored by the A.F. Office of Scientific Research (SREM), 1400 Wilson Boulevard Arlington, Virginia 22209, through the European Office of Aerospace Research, OAR, United States Air Force, under contract F 61052-69-C-0030. 
by the author $[3,6]$. In this work, exact and complete solutions were obtained for problems of vibration and stability of systems of adhering orthotropic layers including the case where the outer layers adhere to one or two elastic half-spaces. The recurrence equations as well as the Thomas-Haskell matrix were evaluated thus providing numerical programming methods. The particular formulation also brings out the fundamental mathematical structure of the problem and the basic physical properties. By putting the initial stress and the frequency equal to zero the results coincide with those obtained from the classical linear elasticity theory, which may be compared with the approximate analysis of the present paper. As far as the single layer is concerned a critical comparison is provided in Table 1. In the case of multilayers the critical feature is adherence where the skin effect plays an essential role. Since it is verified that the approximate treatment includes this effect with good accuracy, it should provide the same accuracy for multilayered systems. Moreover the number of unknowns in the recurrence equations of the approximate theory are reduced by a factor two, while at the same time the coefficients in these equations are quite drastically simplified as illustrated in the particular case of equation (4.6).

There have been numerous attempts in the past to treat problems of multilayered plates on the basis of the classical Euler-Bernouilli assumption. One such treatment [7] considers the most general case of anisotropy. However as shown by Table 1 the EulerBernouilli assumption has restricted validity for anisotropic plates. More recently an analysis based on elasticity theory has been developed [8] for the static problem with anisotropic layers elastically symmetric relative to their own plane. The case of three dimensional deformation based on elasticity theory has been treated for orthotropic layers with the same directions of elastic symmetry [9].

It should be added that this paper is intended only as an introduction. The method has been applied to problems of vibrations of elastic and viscoelastic multilayered plates including the effect of end conditions distributed across the thickness. Furthermore the plane strain solutions are immediately applicable to three-dimensional problems of transverse isotropic materials with rectangular, triangular and circular plan form. These results are presented in a companion paper to be published subsequently.

\section{BASIC EQUATIONS AND ASSUMPTIONS}

We consider an elastic plate of thickness $h$. The $x$ axis is equidistant from the two faces and the $y$ axis is normal to the faces. $\Lambda$ s a first step in the investigation, a plane strain deformation is assumed. The displacement components in the $x y$ plane are denoted by

$$
\begin{aligned}
& u=u(x \cdot y) \\
& v=v(x \cdot y) .
\end{aligned}
$$

The elastic material is orthotropic and the stress-strain relations for plane strain are

$$
\begin{aligned}
\sigma_{x x} & =C_{11} e_{x x}+C_{12} e_{y y} \\
\sigma_{y y} & =C_{12} e_{x x}+C_{22} e_{y y} \\
\sigma_{x y} & =2 L e_{x y}
\end{aligned}
$$


where the strain components are given by

$$
e_{x x}=\frac{\partial u}{\partial x} \quad e_{y y}=\frac{\partial v}{\partial y} \quad 2 e_{x y}=\frac{\partial v}{\partial x}+\frac{\partial u}{\partial y} .
$$

It is also assumed that the material is stratified in such a way that the elastic moduli $C_{11}(y) C_{22}(y) C_{12}(y)$ and $L(y)$ are functions of $y$ only. The corresponding stresses satisfy the following equilibrium conditions

$$
\begin{aligned}
& \frac{\partial \sigma_{x x}}{\partial x}+\frac{\partial \sigma_{x y}}{\partial y}=0 \\
& \frac{\partial \sigma_{x y}}{\partial x}+\frac{\partial \sigma_{y y}}{\partial y}=0 .
\end{aligned}
$$

A fundamental characteristic feature of the mechanics of plates is the smallness of the stress components $\sigma_{y y}$ normal to the faces. Hence we shall put

$$
\sigma_{y y}=0
$$

as a basic approximation in the stress-strain relations (2.2). Thus they become

$$
\begin{aligned}
& \sigma_{x x}=4 M e_{x x} \\
& \sigma_{x y}=2 L e_{x y}
\end{aligned}
$$

with a new modulus.

$$
M=\frac{1}{4 C_{22}}\left(C_{11} C_{22}-C_{12}^{2}\right) \text {. }
$$

In the particular case of an isotropic material in plane strain, the stress-strain relations (2.2) are

$$
\begin{aligned}
\sigma_{x x} & =(\lambda+2 \mu) e_{x x}+\lambda e_{y y} \\
\sigma_{y y} & =\lambda e_{x x}+(\lambda+2 \mu) e_{y y} \\
\sigma_{x y} & =2 \mu e_{x y}
\end{aligned}
$$

where $\lambda$ and $\mu$ are the Lamé constants. In this case we find

$$
\begin{aligned}
M & =\mu \frac{\lambda+\mu}{\lambda+2 \mu} \\
L & =\mu .
\end{aligned}
$$

In terms of Young's modulus $E$ and Poisson's ratio $v$, Lamé's constants are written

$$
\lambda=\frac{E v}{(1+v)(1-2 v)} \quad \mu=\frac{E}{2(1+v)} .
$$

Hence,

$$
M=\frac{1}{4} \frac{E}{1-v^{2}} \quad L=\frac{E}{2(1+v)} .
$$


It is of interest to point out that for an incompressible isotropic material $v=\frac{1}{2}$ and the values (2.11) become

$$
M=L=\frac{E}{3}
$$

Under these conditions the following results are derived. Elimination of $u$ between the two stress-strain relations $(2.6)$ yields

$$
\frac{1}{L} \frac{\partial \sigma_{x y}}{\partial x}-\frac{\partial}{\partial y}\left(\frac{\sigma_{x x}}{4 M}\right)=\frac{\partial^{2} v}{\partial x^{2}}
$$

Further elimination of $\sigma_{x x}$ between this equation and the first equilibrium condition (2.4) leads to

$$
\frac{\partial}{\partial y}\left[\frac{1}{4 M} \frac{\partial \sigma_{x y}}{\partial y}\right]+\frac{1}{L} \frac{\partial^{2} \sigma_{x y}}{\partial x^{2}}=\frac{\partial^{3} v}{\partial x^{3}} .
$$

In the present phase of the analysis we shall consider deformations which are sinusoidally distributed along $x$. Hence we put

$$
\begin{aligned}
u & =U(y) \sin l x \\
v & =V^{\prime}(y) \cos l x \\
\sigma_{x y} & =\tau(y) \sin l x .
\end{aligned}
$$

Equation (2.14) becomes

$$
\frac{\mathrm{d}}{\mathrm{d} y}\left[\frac{1}{4 M} \frac{\mathrm{d} \tau}{\mathrm{d} y}\right]-\frac{l^{2}}{L} \tau=l^{3} V^{\prime}
$$

As an additional assumption we replace $V^{1}$ by a constant $V$ equal to its average across the thickness $h$ of the plate, i.e.

$$
V=\frac{1}{h} \int_{-h / 2}^{h / 2} V^{\prime}(y) \mathrm{d} y .
$$

Hence equation (2.16) is simplified to

$$
\frac{\mathrm{d}}{\mathrm{d} y}\left[\frac{1}{4 M} \frac{\mathrm{d} \tau}{\mathrm{d} y}\right]-\frac{l^{2}}{L} \tau=l^{3} V
$$

This result is fundamental and provides a complete solution of the plate behavior as follows. The shearing stress $\tau(y)$ is obtained by integrating equation (2.20) assuming the boundary values of $\tau$ at the faces $(y= \pm h / 2)$ to be given as applied shear forces. This determines the function $\tau(y)$ in terms of a still unknown parameter $V$. We now integrate the second equilibrium condition (2.1) across the thickness and obtain

$$
\left[\sigma_{y y}\right]_{1}-\left[\sigma_{y y}\right]_{2}=-\int_{-h / 2}^{h / 2} \frac{\partial \sigma_{x y}}{\partial x} \mathrm{~d} y=q \cos l x
$$

where

$$
q=-l \int_{-h / 2}^{h / 2} \tau(y) \mathrm{d} y .
$$


The expression $\left[\sigma_{y y}\right]_{1}-\left[\sigma_{y y}\right]_{2}$ represents the difference of the normal stresses applied at the faces. Hence $q$ is the amplitude of the total normal load applied to the plate per unit area and directed along $y$. When this normal load is known the deflection $V$ is determined by equation (2.22) hence also the distribution $\tau(y)$ of the shear stress. The remaining unknowns are then easily derived. The first equilibrium condition (2.4) yields

$$
\sigma_{x x}=\frac{1}{l} \frac{\mathrm{d} \tau}{\mathrm{d} y} \cos l x
$$

Substitution of this value into the first stress strain relation (2.6) further yields

$$
4 M l^{2} U=\frac{\mathrm{d} \tau}{\mathrm{d} y}
$$

which determines the $x$ component $u=U(y) \sin l x$ of the displacement. The $y$ component of the displacement may be assumed constant across the thickness in first approximation and equal to $v=V \cos l x$.

\section{Thinly laminated material}

The foregoing results are applicable to a plate made of thin alternating layers. For one layer the elastic coefficients are $M_{1}$ and $L_{1}$ and for the other $M_{2}$ and $L_{2}$. These layers occupy respectively fractions $\alpha_{1}$ and $\alpha_{2}$ of the total thickness. If the layers are sufficiently thin the material behaves as an equivalent homogeneous anisotropic medium with elastic coefficients $M$ and $L$. For the case of an incompressible medium they were derived earlier $[2,3]$. However in the present case the procedure of derivation is identical, and the result is formally the same. The equivalent coefficients are

$$
\begin{gathered}
M=M_{1} \alpha_{1}+M_{2} \alpha_{2} \\
L=\frac{L_{1} L_{2}}{L_{2} \alpha_{1}+L_{1} \alpha_{2}} .
\end{gathered}
$$

For a plate of laminated material we may apply equations (2.20) and (2.21) with the values (2.25) for $M$ and $L$. The same procedure is applicable to a laminated medium composed of a repeated sequence of $n$ thin layers each of which occupies a fraction $\alpha_{i}$ of the total thickness and is characterized by the two coefficients $M_{i}$ and $L_{i}$. The equivalent coefficients of the laminated medium are then given by [3],

$$
\begin{aligned}
M & =\sum^{i} M_{i} \alpha_{i} \\
\frac{1}{L} & =\sum^{i} \frac{\alpha_{i}}{L_{i}}
\end{aligned}
$$

The equivalent coefficients (2.26) constitute of course a first approximation. The next approximation is provided in Section 6 below by introducing stress couples. The limits of validity of these concepts were brought out earlier [4] in the context of stability problems taking into account the additional feature of interstitial flow which enters into play for large rigidity contrast between layers. However for the plate bending the equivalent continuum with eventual addition of stress couples should be satisfactory in practice. 


\section{ANALYSIS OF MULTILAYERED PLATES}

The foregoing analysis may be applied to a multilayered plate. This includes the case where some of the layers may be constituted by thinly laminated materials. Within each layer the coefficients $M$ and $L$ are constant. Equation (2.20) is therefore applied to each layer separately while $V$ is assumed to be the same for all layers. For $M$ and $L$ constant, equation (2.20) is readily integrated.

The general integral is

$$
\tau=C_{1} \cosh \beta l y+C_{2} \sinh \beta l y-l L V
$$

with

$$
\beta=2 \sqrt{\frac{M}{L}}
$$

and two integration constants $C_{1}$ and $C_{2}$.

The $x$ axis is assumed to be equally distant from the two boundaries of the layer. Denoting by $\tau_{1}$ and $\tau_{2}$, the values of $\tau$ at the top and bottom of the layer, we derive

$$
\begin{aligned}
C_{1} & =\left[\frac{1}{2}\left(\tau_{1}+\tau_{2}\right)+l L V\right] \frac{1}{\cosh \beta \gamma} \\
C_{2} & =\frac{1}{2}\left(\tau_{1}-\tau_{2}\right) \frac{1}{\sinh \beta \gamma}
\end{aligned}
$$

where

$$
\gamma=\frac{1}{2} l h
$$

and $h$ is the layer thickness.

The normal stresses at the top and bottom boundary produce a total normal loading on the layer given by equation (2.22). Substitution of expressions (3.1) and (3.3) in this equation yields

$$
q=-\left(\tau_{1}+\tau_{2}\right) c+l^{2} h L V\left(1-\frac{c}{\gamma}\right)
$$

with

$$
c=\frac{1}{\beta} \tanh \beta \gamma .
$$

The displacement amplitude $U$ is given by equation (2.24). Its values $U_{1}$ and $U_{2}$ at the top and bottom of the layer are found to be

$$
\begin{aligned}
& U_{1}=\frac{1}{4 l \sqrt{ }(M L)}\left(\tau_{1} a+\tau_{2} b\right)+c V \\
& U_{2}=-\frac{1}{4 l \sqrt{ }(M L)}\left(\tau_{1} b+\tau_{2} a\right)-c V
\end{aligned}
$$


with

$$
\begin{aligned}
& a=\tanh \beta \gamma+\operatorname{cotanh} \beta \gamma \\
& b=\tanh \beta \gamma-\operatorname{cotanh} \beta \gamma .
\end{aligned}
$$

Consider now a plate composed of $n$ orthotropic homogeneous layers. The layers are

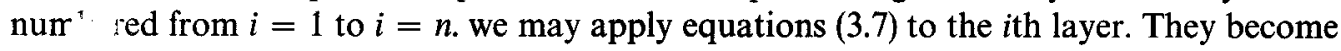

$$
\begin{gathered}
U_{i} l=\frac{1}{4 \sqrt{ }\left(M_{i} L_{i}\right)}\left(\tau_{i} a_{i}+\tau_{i+1} b_{i}\right)+c_{i} l V \\
U_{i+1} l=-\frac{1}{4 \sqrt{ }\left(M_{i} L_{i}\right)}\left(\tau_{i} b_{i}+\tau_{i+1} a_{i}\right)-c_{i} l V .
\end{gathered}
$$

In these relations $V$ now represents the average value of the normal displacement across the whole multilayered plate. The elastic coefficients of the $i$ th layer are $M_{i}$ and $L_{i}$ and its thickness is $h_{i}$. The values of $a_{i}, b_{i}, c_{i}$ are obtained by substituting $\beta_{i}=2 \sqrt{(}\left(M_{i} / L_{i}\right)$ and $\gamma_{i}=\left(\frac{1}{2}\right) l h_{i}$ into expressions (3.6) and (3.8) for $a, b, c$.

We may write equations (3.9) in abbreviated form by putting

with

$$
\mathscr{T}_{i}=\frac{1}{2} A_{i} \tau_{i}^{2}+B_{i} \tau_{i} \tau_{i+1}+\frac{1}{2} A_{i} \tau_{i+1}^{2}
$$

$$
A_{i}=\frac{a_{i}}{4 \sqrt{ }\left(M_{i} L_{i}\right)} \quad B_{i}=\frac{b_{i}}{4 \sqrt{ }\left(M_{i} L_{i}\right)}
$$

Equations (3.9) become

$$
\begin{gathered}
U_{i} l=\frac{\partial \mathscr{T}_{i}}{\partial \tau_{i}}+c_{i} l V \\
U_{i+1} l=-\frac{\partial \mathscr{T}_{i}}{\partial \tau_{i+1}}-c_{i} l V .
\end{gathered}
$$

At the interfaces between the layers $i$ and $i+1$ the displacement $U_{i+1}$ is the same when considered as belonging to layer $i$ or $i+1$. According to equations (3.12) this is expressed by the relations

$$
-\frac{\partial \mathscr{T}_{i}}{\partial \tau_{i+1}}-c_{i} l V=\frac{\partial \mathscr{T}_{i+1}}{\partial \tau_{i+1}}+c_{i+1} l V
$$

or

$$
B_{i} \tau_{i}+\left(A_{i}+A_{i+1}\right) \tau_{i+1}+B_{i+1} \tau_{i+2}=-\left(c_{i}+c_{i+1}\right) l V .
$$

Thus we have obtained $n-1$ recurrence equations for the values of $\tau_{i}$. Hence when the values $\tau_{1}$ and $\tau_{n+1}$ are given at the outerfaces of the multilayered plate the values of $\tau_{i}$ at the $n-1$ interfaces are obtained in terms of $V$ by solving the $n-1$ recurrence equations (3.14). For example if the shear stresses $\tau_{1}$ and $\tau_{n+1}$ at the outerfaces are zero the general expression for $\tau_{j}$ may be written

$$
\tau_{j}=D_{j} l V
$$

where $D_{1}=D_{n+1}=0$. 
Next we shall apply equation (3.5) to the $i$ th layer. The normal load $q_{i}$ acting on this layer is

$$
q_{i}=-\left(\tau_{i}+\tau_{i+1}\right) c_{i}-2 L_{i} c_{i} l V+L_{i} h_{i} l^{2} V .
$$

Substitution of the value (3.15) for $\tau_{j}$ yields

$$
q_{i}=\left[l h_{i} L_{i}-\left(D_{i}+D_{i+1}\right) c_{i}-2 L_{i} c_{i}\right] l V .
$$

The total normal load $q$ applied to the multilayered plate is obtained by summation of the loads $q_{i}$ applied to each layer. We obtain

$$
q=\sum_{i=1}^{n} q_{i}
$$

or

$$
q=l V \sum_{i=1}^{n}\left[l h_{i} L_{i}-\left(D_{i}+D_{i+1}\right) c_{i}-2 L_{i} c_{i}\right] .
$$

This result yields the normal deflection $V$ under a given normal loading. The shear stresses at the interfaces are then determined by equations (3.15).

\section{THE HOMOGENEOUS ANISOTROPIC PLATE}

We shall apply the foregoing results to the single homogeneous anisotropic plate of thickness $h$. The shear stresses applied to the faces are assumed to be zero. Hence putting $\tau_{1}=0$ and $\tau_{2}=0$ in equation (3.5) we derive the normal load

$$
q=\left(1-\frac{\tanh \beta \gamma}{\beta \gamma}\right) l^{2} h L V
$$

For wavelengths large compared to the plate thickness, i.e. for small values of $l$ we replace $\tanh \beta \gamma$ by the first two terms of its Taylor expansion. This yields

$$
q=\frac{1}{3} M h^{3} l^{4} V .
$$

For an isotropic material of Young's modulus $E$ and Poisson's ratio $v$ this becomes

$$
q=\frac{1}{12} \frac{E}{1-v^{2}} h^{3} l^{4} V .
$$

Expressions (4.2) and (4.3) coincide with the results obtained from the classical EulerBernouilli theory of plates where the cross-section is assumed to remain plane and normal to the neutral axis.

It is of interest to compare the approximate result (4.1) which we write in the form

$$
\frac{q}{l^{2} h L V}=1-\frac{\tanh \beta \gamma}{\beta \gamma}
$$

with the exact value of the same quantity derived from the theory of Elasticity for the case of an incompressible material. This exact value obtained previously $[2,3]$ is

$$
\frac{q}{l^{2} h L V}=\frac{A}{\gamma}
$$


where

$$
\begin{aligned}
A & =\frac{\left(\beta_{1}^{2}+1\right)^{2} \beta_{2}}{\beta_{1}^{2}-\beta_{2}^{2}} \tanh \beta_{2} \gamma-\frac{\left(\beta_{2}^{2}+1\right)^{2} \beta_{1}}{\beta_{1}^{2}-\beta_{2}^{2}} \tanh \beta_{1} \gamma \\
\beta_{1} & =\sqrt{ }\left[m+\sqrt{ }\left(m^{2}-1\right)\right] \\
\beta_{2} & =\sqrt{ }\left[m-\sqrt{ }\left(m^{2}-1\right)\right] \\
m & =\frac{2 M}{L}-1 .
\end{aligned}
$$

We may also compare these results with the value given by the Euler-Bernouilli theory which is obtained by retaining the first two terms of the Taylor expansion of $\tanh \beta \gamma$ in equation (4.4) namely

$$
\frac{q}{l^{2} h L V}=\frac{1}{3}(\beta \gamma)^{2} .
$$

The numerical values of expressions (4.4), (4.5) and (4.7) are shown in Table 1 for a number of cases.

TABLE 1

\begin{tabular}{ccccl}
\hline$M / L$ & $\gamma$ & $1-(\tanh \beta \gamma / \beta \gamma)$ & $A / \gamma$ & $\frac{1}{3}(\beta \gamma)^{2}$ \\
\hline 1 & 0.3 & 0.107 & 0.114 & 0.120 \\
2.2 & 0.3 & 0.203 & 0.217 & 0.270 \\
2.2 & 0.6 & 0.474 & 0.532 & 1.08 \\
9 & 0.3 & 0.474 & 0.495 & 1.08 \\
9 & 0.6 & 0.714 & 0.750 & 4.32 \\
\hline
\end{tabular}

The value $\gamma=0.3$ corresponds to a wavelength about ten times the thickness $h$. For $\gamma=0.6$ the wavelength is about five times the thickness. We note that the error of the EulerBernouilli value is large except for the case $M / L=1$ corresponding to an isotropic incompressible material. However the approximate value (4.4) remains adequate.

We may verify that the accuracy of the approximate value (4.4) increases with increasing anisotropy. This is shown by evaluating the limiting value of the exact expression (4.5) for large values of $M / L$. In this case we write approximately

$$
\beta_{1} \cong \beta \quad \beta_{2} \cong \frac{1}{\beta}
$$

Assuming that $\gamma$ is restricted to the range

$$
\gamma<\beta / 10
$$

the exact value (4.5) tends to

$$
\frac{A}{\gamma} \cong 1-\frac{\tanh \beta \gamma}{\beta \gamma}
$$

which is the same as the approximation (4.4). 
It is also of interest to point out that in the limiting case of very large anisotropy and for wavelengths which are not too large, expression (4.4) may be replaced by unity. Hence in this limiting case

$$
q=l^{2} h L V .
$$

Physically this corresponds to a plate deflecting in pure shear.

\section{THE TWO- AND THREE-LAYERED PLATE}

Consider a plate composed of two layers. The material of the first layer of thickness $h_{1}$ is elastic and orthotropic of elastic constants $M_{1}$ and $L_{1}$. The thickness and elastic constant of the second layer are denoted by $h_{2}, M_{2}$ and $L_{2}$. The total thickness of the plate is $h=h_{1}+h_{2}$. The applied shear stresses at the outerfaces are put equal to zero

$$
\tau_{1}=\tau_{3}=0 \text {. }
$$

The shear stress $\tau_{2}$ at the interface is found by equating the interfacial displacements $U_{2}$ considered as belonging to the first and second layer. Applying equations (3.7) we derive

$$
\begin{aligned}
& U_{2} l=\frac{1}{4 \sqrt{\left(M_{2} L_{2}\right)}} \tau_{2} a_{2}+c_{2} l V \\
& U_{2} l=-\frac{1}{4 \sqrt{\left(M_{1} L_{1}\right)}} \tau_{2} a_{1}-c_{1} l V .
\end{aligned}
$$

Equating the two values of $U_{2}$ yields

$$
\tau_{2}=-\frac{4\left(c_{1}+c_{2}\right)}{a_{1} / \sqrt{ }\left(M_{1} L_{1}\right)+a_{2} / \sqrt{ }\left(M_{2} L_{2}\right)} l V .
$$

In this expression the values of $a_{1}$ and $c_{2}$ are obtained from equations (3.6) and (3.8) substituting $M_{1}, L_{1}$ and $h_{1}$ for $M, L$ and $h$. Similarly $a_{2}$ and $c_{2}$ are obtained by substituting $M_{2}, L_{2}$ and $h_{2}$ in the same equations.

The total normal load $q$ is the sum of the normal loads $q_{1}$ and $q_{2}$ on each layer

$$
q=q_{1}+q_{2} \text {. }
$$

Using equation (3.5) after substituting the value (5.3) of $\tau_{2}$ we obtain

$$
\frac{q}{l V}=\frac{4\left(c_{1}+c_{2}\right)^{2}}{a_{1} / \sqrt{ }\left(M_{1} L_{1}\right)+a_{2} / \sqrt{ }\left(M_{2} L_{2}\right)}+l\left(L_{1} h_{1}+L_{2} h_{2}\right)-2\left(L_{1} c_{1}+L_{2} c_{2}\right) .
$$

This determines the deflection $V$ when the normal load and the wavelength are given.

Consider now a threelayered plate composed of a layer of thickness $h_{2}$ and elastic coefficients $M_{2}, L_{2}$ sandwiched between two layers of thickness $h_{1}$ and elastic coefficients $M_{1}, L_{1}$. Because of the symmetry, the interfacial shears are $\tau_{2}=\tau_{3}$ while $\tau_{1}=\tau_{4}=0$ at the outerfaces. Using relations (3.7) we equate the interfacial displacements $U_{2}$ considered as belonging to layers 1 and 2 . We obtain

$$
-\frac{1}{4 \sqrt{ }\left(M_{1} L_{1}\right)} \tau_{2} a_{1}-c_{1} l V=\frac{1}{4 \sqrt{ }\left(M_{2} L_{2}\right)} \tau_{2}\left(a_{2}+b_{2}\right)+c_{2} l V .
$$


Solving for $\tau_{2}$, taking into account the identity $a_{2}+b_{2}=2 \beta_{2} c_{2}$, yields

$$
\tau_{2}=-\frac{4\left(c_{1}+c_{2}\right)}{a_{1} / \sqrt{ }\left(M_{1} L_{1}\right)+2 \beta_{2} c_{2} /\left(\sqrt{ } M_{2} L_{2}\right)} l V .
$$

According to equation (3.18) the total normal load is

$$
q=2 q_{1}+q_{2} \text {. }
$$

By applying equation (3.5) with $\tau_{1}=\tau_{4}=0$ and the value (5.7) for $\tau_{2}=\tau_{3}$ we derive

$$
\frac{q}{l V}=\frac{8\left(c_{1}+c_{2}\right)^{2}}{a_{1} / \sqrt{ }\left(M_{1} L_{1}\right)+2 \beta_{2} c_{2} / \sqrt{ }\left(M_{2} L_{2}\right)}+2 l h_{1} L_{1}\left(1-\frac{c_{1}}{\gamma_{1}}\right)+l h_{2} L_{2}\left(1-\frac{c_{2}}{\gamma_{2}}\right) .
$$

The last two terms represent the normal load corresponding to superposed layers with perfect interfacial slip. The first term represents the effect of adherence between layers. An interesting result is obtained for the limiting case of large wavelength, i.e. for small values of $\gamma_{1}$ and $\gamma_{2}$. In this case equation (5.9) becomes

$$
q=\left[2\left(h_{1}+h_{2}\right)^{2} h_{1}+\frac{2}{3} h_{1}^{3}\right] M_{1} l^{4} V+\frac{1}{3} M_{2} h_{2}^{3} l^{4} V .
$$

This is the same as obtained by assuming the cross-section to remain plane and normal to the neutral axis.

\section{EXTENSION TO THINLY LAMINATED MEDIA WITH STRESS COUPLES}

We consider a laminated medium constituted by an alternation of thin layer of thickness $h_{1}=a_{1} h^{\prime}$ and $h_{2}=\alpha_{2} h^{\prime}$. The total thickness of a pair of layers is $h^{\prime}=h_{1}+h_{2}$. Their elastic coefficients are denoted by $M_{1}, L_{1}$ and $M_{2}, L_{2}$, respectively. In some cases use of the equivalent anisotropic continuum with elastic coefficients (2.25) will not be sufficient and the bending rigidity of the laminations must be taken into account. This can be done by introducing stress couples as follows.

We first evaluate the bending moment $\mathscr{M}_{1}$ in the layer of thickness $h_{1}$. We locate the $x$ axis midway between the boundaries of the layer and write

$$
\mathscr{M}_{1}=-\int_{-h_{1} / 2}^{h_{1} / 2} \sigma_{x x} y \mathrm{~d} y .
$$

The minus sign is chosen by defining $\mathscr{M}_{1}$ as the couple obtained by reversing the stress $\sigma_{x x}$. The value (2.23) for $\sigma_{x x}$ is introduced using expressions (3.1) and (3.3) for $\tau$. This yields

$$
\mathscr{M}_{1}=-\frac{h_{1}}{l}\left[\frac{1}{2}\left(\tau_{1}+\tau_{2}\right)+l L_{1} V\right]\left(1-\frac{\tanh \beta_{1} \gamma_{1}}{\beta_{1} \gamma_{1}}\right) \cos l x
$$

where $\tau_{1}$ and $\tau_{2}$ are the shear stresses at the top and bottom of the layer, while $\beta_{1}=$ $2 \sqrt{ }\left(M_{1} / L_{1}\right)$ and $\gamma_{1}=\frac{1}{2} l h_{1}$. There are several important simplifications which may be introduced at this stage. We note that $\frac{1}{2}\left(\tau_{1}+\tau_{2}\right) \sin l x=\sigma_{x y}$ represents the average shear stress of the equivalent continuum. Hence we may write

$$
\frac{1}{2}\left(\tau_{1}+\tau_{2}\right) \sin l x=2 L e_{x y}
$$


where $e_{x y}$ is the average shear strain and $L$ is given by equations (2.25). On the other hand, the case where the plate remains flat should produce only negligible stress couples. Therefore we need only take into account the normal displacement $V$. As a consequence we may substitute the value

$$
2 e_{x y}=\frac{\partial v}{\partial x}=-l V \sin l x
$$

Hence from (6.3)

$$
\frac{1}{2}\left(\tau_{1}+\tau_{2}\right)=-L l V .
$$

Another simplication is obtained by using the approximation

$$
1-\frac{\tanh \beta_{1} \gamma_{1}}{\beta_{1} \gamma_{1}}=\frac{1}{3} \beta_{1}^{2} \gamma_{1}^{2}=\frac{1}{3} \frac{M_{1}}{L_{1}} h_{1}^{2} l^{2}
$$

which is valid for a thin layer if $\beta_{1} \gamma_{1} \ll 1$.

Finally we may also put

$$
\frac{\partial^{2} v}{\partial x^{2}}=-l^{2} V \cos l x
$$

With the values (6.5)-(6.7) the bending moment (6.2) becomes

$$
\mathscr{M}_{1}=\frac{1}{3}\left(L_{1}-L\right) \frac{M_{1}}{L_{1}} h_{1}^{3} \frac{\partial^{2} v}{\partial x^{2}}
$$

Introducing the value (2.25) for $L$ and putting $h_{1}=\alpha_{1} h^{\prime}$ we obtain

$$
\mathscr{M}_{1}=\frac{1}{3} h^{\prime 3} \frac{\left(L_{1}-L_{2}\right)}{\alpha_{1} L_{2}+\alpha_{2} L_{1}} M_{1} \alpha_{2} \alpha_{1}^{3} \frac{\partial^{2} v}{\partial x^{2}}
$$

Similarly for the layer of thickness $h_{2}$ the bending moment is

$$
\mathscr{M}_{2}=\frac{1}{3} h^{\prime 3} \frac{\left(L_{2}-L_{1}\right)}{\alpha_{1} L_{2}+\alpha_{2} L_{1}} M_{2} \alpha_{1} \alpha_{2}^{3} \frac{\partial^{2} v}{\partial x^{2}} .
$$

The total stress couple per unit thickness is therefore

$$
\mathscr{M}=\frac{1}{h^{\prime}}\left(\mathscr{M}_{1}+\mathscr{M}_{2}\right)=b \frac{\partial^{2} v}{\partial x^{2}}
$$

with the stress couple coefficient

$$
b=\frac{1}{3} h^{\prime 2} \alpha_{1} \alpha_{2} \frac{\left(L_{1}-L_{2}\right)}{\alpha_{1} L_{2}+\alpha_{2} L_{1}}\left(M_{1} \alpha_{1}^{2}-M_{2} \alpha_{2}^{2}\right) .
$$

This result coincides with the value derived previously by a different method for the particular case of an incompressible material [4].

If one of the layers is very stiff compared to the other $M_{1} \gg M_{2}, L_{1} \gg L_{2}$ the value (6.12) is simplified to

$$
b=\frac{1}{3} h_{1}^{2} M
$$

where $M=\alpha_{1} M_{1}$ represents an elastic coefficient of the equivalent continuum. 
The result is easily extended to the case where the laminated medium is composed of repeated groups of $n$ thin layers. Each layer is denoted by the subscript $i$. The total thickness of the group being $h^{\prime}$ the thickness of each layer is $h_{i}=\alpha_{i} h^{\prime}$. Expressions such as (6.8) may be used for the bending moment $\mathscr{M}_{i}$ in each layer. The total stress couple per unit thickness is therefore

$$
\mathscr{M}=\frac{1}{h^{\prime}} \sum \mathscr{M}_{i}=b \frac{\partial^{2} v}{\partial x^{2}}
$$

where

$$
b=\frac{1}{3} h^{\prime 2} \sum^{i}\left(1-\frac{L}{L_{i}}\right) M_{i} \alpha_{i}^{3}
$$

and $L$ is the coefficient of the equivalent continuum given by equation (2.26).

We shall now cxamine how the basic cquations of Sections 2 and 3 must be modified in order to take into account the couple stresses. The only change is in the second equilibrium equation (2.4) which is now replaced by

$$
\frac{\partial \sigma_{y x}}{\partial x}+\frac{\partial \sigma_{y y}}{\partial y}=0
$$

with

$$
\sigma_{x y}-\sigma_{y x}=\frac{\partial \mathscr{M}}{\partial x} .
$$

Elimination of $\sigma_{y x}$ between these two equations yields

$$
\frac{\partial \sigma_{x y}}{\partial x}+\frac{\partial \sigma_{y y}}{\partial y}=\frac{\partial^{2} \mathscr{M}}{\partial x^{2}}=b \frac{\partial^{4} v}{\partial x^{4}}
$$

For a laminated layer of thickness $h$ and constant coefficients the value of $q$ is obtained as in equation (2.2) by integrating the equilibrium equation (6.18) across the thickness. We obtain

$$
q=-l \int_{-h / 2}^{h / 2} \tau(y) \mathrm{d} y+b h l^{4} V
$$

Since the value (3.1) of $\tau$ remains the same

$$
q=-\left(\tau_{1}+\tau_{2}\right) c+l^{2} h L V\left(1-\frac{c}{\gamma}\right)+b h l^{4} V .
$$

For a multilayered plate where the various layers may be laminated each with a stress couple-coefficient $b_{i}$ the value (3.19) is replaced by

$$
q=l V \sum_{i=1}^{n}\left[l h_{i} L_{i}-\left(D_{i}+D_{i+1}\right) c_{i}-2 L_{i} c_{i}\right]+l^{4} V \sum_{i=1}^{n} b_{i} h_{i} .
$$

All other equations remain unchanged and the analysis follows exactly the same procedure as in Section 3. 


\section{DISCUSSION OF CHARACTERISTIC FEATURES}

Consider the case of the homogeneous anisotropic plate discussed in Section 4 . The shearing stress distribution is obtained by putting $\tau_{1}=\tau_{2}=0$ in equations (3.1) and (3.3). We find

$$
\tau=\left(\frac{\cosh \beta l y}{\cosh \frac{1}{2} \beta l h}-1\right) l L V .
$$

Substitution of this value in equation (2.23) yields the corresponding bending stress distribution.

$$
\sigma_{x x}=\beta \frac{\sinh \beta l y}{\cosh \frac{1}{2} \beta l h} l L V \cos l x .
$$

For large wavelengths, $l$ is small, and for a given value of $\beta$ the distributions (7.1) and (7.2) tend to the limits

$$
\begin{aligned}
\tau & =2 M\left(y^{2}-\frac{h^{2}}{4}\right) l^{3} V \\
\sigma_{x x} & =4 M y l^{2} V \cos l x .
\end{aligned}
$$

These linear and parabolic distributions are the same as derived from the classical theory of thin plates where the deflection $v$ under a load distribution $q(x)$ is governed by the differential equation

$$
\frac{1}{3} M h^{3} \frac{\mathrm{d}^{4} v}{\mathrm{~d} x^{4}}=q(x) .
$$

The deflection $V$ for a sinusoidal load in this limiting case is given by equation (4.2).

Consider now the other extremc where $l$ is fixed while the anisotropy increases indefinitely, hence for $\beta \rightarrow \infty$. In this case the deflection for a sinusoidal loading is expressed by equation (4.11) which corresponds to the differential equation

$$
\operatorname{Lh} \frac{\mathrm{d}^{2} v}{\mathrm{~d} x^{2}}=-q(x) .
$$

It represents a plate deforming in pure shear. The value (7.1) of $\tau$ is then almost constant over the cross-section and equal to

$$
\tau=-l L V
$$

except for a thin region near the boundaries where it rapidly drops to zero. Similarly the value (7.2) of the bending stress $\sigma_{x x}$ is near zero except in the same thin region where (at the cross-section $x=0$ ) it rapidly rises to the value

$$
\sigma_{\max }=\beta l L V
$$

at the boundary.

These results coincide with the existence of a fundamental skin effect derived and discussed in a previous paper [1]. The thickness of the boundary layer corresponding to this 
The result is easily extended to the case where the laminated medium is composed of repeated groups of $n$ thin layers. Each layer is denoted by the subscript $i$. The total thickness of the group being $h^{\prime}$ the thickness of each layer is $h_{i}=\alpha_{i} h^{\prime}$. Expressions such as (6.8) may be used for the bending moment $\mathscr{M}_{i}$ in each layer. The total stress couple per unit thickness is therefore

$$
\mathscr{M}=\frac{1}{h^{\prime}} \sum^{i} \mathscr{M}_{i}=b \frac{\partial^{2} v}{\partial x^{2}}
$$

where

$$
b=\frac{1}{3} h^{\prime 2} \sum^{i}\left(\begin{array}{ll}
1 & L \\
L_{i}
\end{array}\right) M_{i} \alpha_{i}^{3}
$$

and $L$ is the coefficient of the equivalent continuum given by equation (2.26).

We shall now examine how the basic equations of Sections 2 and 3 must be modified in order to take into account the couple stresses. The only change is in the second equilibrium equation (2.4) which is now replaced by

$$
\frac{\partial \sigma_{y x}}{\partial x}+\frac{\partial \sigma_{y y}}{\partial y}=0
$$

with

$$
\sigma_{x y}-\sigma_{y x}=\frac{\partial \mathscr{M}}{\partial x}
$$

Elimination of $\sigma_{y x}$ between these two equations yields

$$
\frac{\partial \sigma_{x y}}{\partial x}+\frac{\partial \sigma_{y y}}{\partial y}=\frac{\partial^{2} \mathscr{M}}{\partial x^{2}}=b \frac{\partial^{4} v}{\partial x^{4}} .
$$

For a laminated layer of thickness $h$ and constant coefficients the value of $q$ is obtained as in equation (2.2) by integrating the equilibrium equation (6.18) across the thickness. We obtain

$$
q=-l \int_{-h / 2}^{h / 2} \tau(y) \mathrm{d} y+b h l^{4} V .
$$

Since the value (3.1) of $\tau$ remains the same

$$
q=-\left(\tau_{1}+\tau_{2}\right) c+l^{2} h L V\left(1-\frac{c}{\gamma}\right)+b h l^{4} V
$$

For a multilayered plate where the various layers may be laminated each with a stress couple-coefficient $b_{i}$ the value (3.19) is replaced by

$$
q=l V \sum_{i=1}^{n}\left[l h_{i} L_{i}-\left(D_{i}+D_{i+1}\right) c_{i}-2 L_{i} c_{i}\right]+l^{4} V \sum_{i=1}^{n} b_{i} h_{i} .
$$

All other equations remain unchanged and the analysis follows exactly the same procedure as in Section 3. 


\section{DISCUSSION OF CHARACTERISTIC FEATURES}

Consider the case of the homogeneous anisotropic plate discussed in Section 4. The shearing stress distribution is obtained by putting $\tau_{1}=\tau_{2}=0$ in equations (3.1) and (3.3). We find

$$
\tau=\left(\frac{\cosh \beta l y}{\cosh \frac{1}{2} \beta l h}-1\right) l L V .
$$

Substitution of this value in equation (2.23) yields the corresponding bending stress distribution.

$$
\sigma_{x x}=\beta \frac{\sinh \beta l y}{\cosh \frac{1}{2} \beta l h} l L V \cos l x .
$$

For large wavelengths, $l$ is small, and for a given value of $\beta$ the distributions (7.1) and (7.2) tend to the limits

$$
\begin{aligned}
\tau & =2 M\left(y^{2}-\frac{h^{2}}{4}\right) l^{3} V \\
\sigma_{x x} & =4 M y l^{2} V \cos l x .
\end{aligned}
$$

These linear and parabolic distributions are the same as derived from the classical theory of thin plates where the deflection $v$ under a load distribution $q(x)$ is governed by the differential equation

$$
\frac{1}{3} M h^{3} \frac{\mathrm{d}^{4} v}{\mathrm{~d} x^{4}}=q(x)
$$

The deflection $V$ for a sinusoidal load in this limiting case is given by equation (4.2).

Consider now the other extreme where $l$ is fixed while the anisotropy increases indefinitely, hence for $\beta \rightarrow \infty$. In this case the deflection for a sinusoidal loading is expressed by equation (4.11) which corresponds to the differential equation

$$
\operatorname{Lh} \frac{\mathrm{d}^{2} v}{\mathrm{~d} x^{2}}=-q(x) .
$$

It represents a plate deforming in pure shear. The value (7.1) of $\tau$ is then almost constant over the cross-section and equal to

$$
\tau=-l L V
$$

except for a thin region near the boundaries where it rapidly drops to zero. Similarly the value (7.2) of the bending stress $\sigma_{x x}$ is near zero except in the same thin region where (at the cross-section $x=0$ ) it rapidly rises to the value

$$
\sigma_{\max }=\beta l L V
$$

at the boundary.

These results coincide with the existence of a fundamental skin effect derived and discussed in a previous paper [1]. The thickness of the boundary layer corresponding to this 
skin effect was found to be

$$
\delta=\frac{1}{\beta l} \text {. }
$$

The same value is also derived from expressions (7.1) and (7.2).

Another characteristic feature is brought out by considering equation (4.1) which provides a relation between the load $q$ and the deflection $V$. For values $z<5$ a suitable approximation is

$$
1-\frac{\tanh z}{z}=\frac{z^{2}}{3+1 \cdot 18 z^{2}} .
$$

Hence equation (4.1) may be written approximately

$$
q=\frac{M h^{3} l^{4}}{3+1 \cdot 18(M / L) l^{2} h^{2}} .
$$

For a given ration $M / L$ and sufficiently large wavelength this value coincides with equation (4.2) obtained from the classical bending theory. However as the wavelength decreases the term $1 \cdot 18(M / L) l^{2} h^{2}$ in the denominator enters into play and the correction is of the order of 10 per cent if

$$
l h \cong \frac{1}{2} \sqrt{\frac{L}{M}}=\frac{1}{\beta} .
$$

This determines a transition wavelength

$$
\mathscr{L}=\frac{2 \pi}{l}=2 \pi \beta h
$$

below which the shear deformation becomes significant. This concept of transition wavelength was already discussed earlier in connection with geological problems of folding instability [5]. It is essentially related to the skin effect as can be seen by writing condition (7.11) in the form

$$
h=\delta .
$$

Hence the transition wavelength is reached where the boundary layer thickness is of the order of the plate thickness. It should be pointed out that for large anisotropy this transition wavelength is not small. For example for $M / L=9$ we find $\mathscr{L}=6 \pi h$ which is about eighteen times the plate thickness.

It is interesting to compare these results with a "Timoshenko beam" approach which may be formulated by the following equations

$$
\begin{array}{ll}
\frac{\mathrm{d} S}{\mathrm{~d} x}+q(x)=0 & \frac{\mathrm{d} \mathscr{M}}{\mathrm{d} x}+S=0 \\
\frac{1}{3} M h^{3} \frac{\mathrm{d} \phi}{\mathrm{d} x}=-\mathscr{M} & \frac{\mathrm{d} v}{\mathrm{~d} x}+\phi=\frac{S}{K h L}
\end{array}
$$

where $q(x)$ is the distributed normal load, $S$ the total cross-sectional shear force, $\mathscr{M}$ the bending moment, $\phi$ the angle of rotation of a cross-section, $v$ the deflection and $K$ a 
dimensionless coefficient. For a sinusoidal load distribution $q(x)=q \cos l x$ and a corresponding deflection $v=V \cos l x$ equations (7.14) yields the relation

$$
q=\frac{M h^{3} l^{4}}{3+M / K L l^{2} h^{2}} V
$$

This coincides with equation (7.10) provided we put

$$
\frac{1}{K}=1 \cdot 18 \text {. }
$$

Hence the present method provides the value of the unknown coefficient $K$ which yields the approximately correct deflection.

However the Timoshenko beam approach ignores the skin effect and does not provide the detailed structure of the stress field or the cross-sectional distortion which must be taken into account for an accurate estimate of failure stresses effective damping and the interaction of adherent layers in multilayered plates.

Characteristic features such as the existence of a transition wavelength and others examined here in the context of the homogeneous anisotropic plate should also be present in the mechanics of multilayered plates even for isotropic materials because of the overall anisotropy of such systems.

A final remark is in order concerning non-sinusoidal load distributions and application to plates of finite span. It is clear that the half wavelength of the sinusoidal solution represents the response of a plate of span $\pi / l$ simply supported at both ends. A load which is not sinusoidal may be expanded in a Fourier series, each component corresponding to a wavelength which is a submultiple of the fundamental. The response of the plate is obtained by applying the present theory to each component. The result will usually converge quite rapidly.

\section{REFERENCES}

[1] M. A. Biot, Fundamental skin effect in anisotropic solid mechanics. Int. J. Solids Struct. 2, 645-663 (1966).

[2] M. A. BIOT, Theory of slability of multilayered continua in finite anisotropic elasticity. J. Franklin Inst. 276, $128-153(1963)$.

[3] M. A. Biot, Mechanics of Incremental Deformations. John Wiley (1965).

[4] M. A. Biot, Rheological stability with couple stresses and its application to geological folding. Proc. R. Soc. A298, 402-423 (1967).

[5] M. A. BIot, Theory of similar folding of the first and second kind. Geol. Soc. Am. Bull. 76, 251-258 (1965).

[6] M. A. Biot, Continuum dynamics of elastic plates and multilayered solids under initial stress. J. Math. Mech. 12, 793-810 (1963).

[7] Y. Stravsk Y, Bending and stretching of aelotropic plates. J. Eng. Mech. Div. Am. Soc. civ. Engrs 87, 31-56 (1961).

[8] N. J. PaGANO, Influence of shear coupling in cylindrical bending of anisotropic laminates. J. Composite Mater. 4, 330-343 (1970).

[9] S. SRINIVAS and A. K. KAO, Bending, vibration and buckling of simply supported thick orthotropic rectangular plates and laminates. Int. J. Solids Struct. 6, 1463-1481 (1970).

(Received 11 March 1971; revised 17 August 1971)

Абстракт-Разработаны новые методы, приводящие к упрощенной трактировке многослойных и сильно ортотропных пластинок. Предлагаемая теория учитывает случай, когда эти отдельныеслои состовляют собой тонко пластинчатые материалы, из которых каждый обладает своими собственными свойствами ортотропии и микроупругости. Учитывается основной "зффект поверхностного слоя" механики анизотропных твердых тел. Выводится теория для случая плоских деформаций. Получаются рекуррентные уравнения, для произвольного числа слоев и применяются $\mathbf{k}$ пластинкам с двумя или тремя слоями. Определяются микроупругие моментные напряжения для случая пласминчатой среды. Обсуждаются характеристические физические свойства. Результаты сравниваются с подходом, основанном на классических моделях Эйлера-Бернулли и Тимошенки. 